\title{
Simon Dalley \\ LIGHTCONE 2011 Summary: applications of Light-Cone Coordinates to Highly Relativistic Systems
}

Received: 16 June 2011 / Accepted: 30 June 2011 / Published online: 13 July 2011

(C) Springer-Verlag 2011

The 2011 workshop of the International Light Cone Advisory Committee (ILCAC), Inc., took place at Southern Methodist University (SMU) in Dallas, Texas, 23-27 May. These workshops have been held at least annually since 1990, focusing on the formal and phenomenological application of light-cone coordinates to nuclear, particle, and string physics, especially via light-front quantization of field theory. In short, the field studies physics from the viewpoint of an observer travelling at the speed of light, a frame of reference that has some unique kinematical and dynamical advantages.

LIGHTCONE 2011 had about 50 physicists, featuring a total of 45 presentations (invited talks and posters). The workshop was purposely limited in size to avoid parallel sessions and to allow all participants to present if they wished. Each day began with a 1 -h thematic keynote talk, followed by $1 / 2 \mathrm{~h}$ talks; a relatively large and well-attended poster session generated a lot of discussion. About half the working time of the conference was given to informal discussion.

The daily themes of this workshop reflected the current interests of the field: AdS/CFT/QCD, Partons, Ab Initio Calculations, Nuclear and Many-Body, with the final morning covering a variety of topics. Presentations have therefore been divided into those themes in this volume. Many phenomenological applications to hadrons were discussed using holographic QCD, covariant constituent models, and chiral perturbation theory. Light-front holography, which maps AdS/QCD directly to LF QCD in physical space-time, was treated in talks by Brodsky and de Teramond. Discussions on the kinematic issues in extracting GPDs (B. Bakker and C. Ji) were highly relevant to the analysis of DVCS experiments at JLab and DESY. A number of new directions were presented at this workshop, including the phenomenology of multi-parton distributions (M. Diehl) and new computational methods, such as Light-cone Coupled-cluster (J. Hiller and S. Chabysheva) and Singular Value Decomposition (M. Weinstein). The most lively discussion centered on 'in-hadron' condensates, which eliminate the 45 orders of magnitude conflict between the cosmological constant and QCD (talks by Roberts, Brodsky, Tandy, Glazek) and reminiscent of the trivial vacuum of regulated light-front quantization. The in-hadron condensate idea, which seems at odds with the traditional idea of Lorentz invariant vacuumexpectation values associated to spontaneous symmetry breaking, was supported by some but vigorously opposed by other participants. Many other interesting presentations can be found in this volume-they are only half the story however, as discussions outside of the lecture room were as important.

Acknowledgments I wish to thank the local organizing committee F. Olness, P. Nadolsky, K. Hornbostel and the session convenors S. Brodsky, M. Burkardt, T. Frederico, J. Vary, for helping with logistics and attracting good speakers and topics, the ILCAC for their supporting a workshop in Dallas, and staff and students of the Physics Department C. Carroll, S. Melton, L. Zhu, F. Ferrante for making things run smoothly. The workshop received financial support from the SMU Dean of Research and Graduate Studies, the SMU Physics Department through a donation from the Lightner-Sams Foundation, Springer-Verlag Publishing, and the Gary McCartor Travel Awards.

S. Dalley $(\varangle)$

Department of Physics, Southern Methodist University, Dallas, TX 75275-0175, USA

E-mail: sdalley@smu.edu 